Für $\mathcal{T e}_{4} 45$ in der Breite $=-11^{\circ}$ :

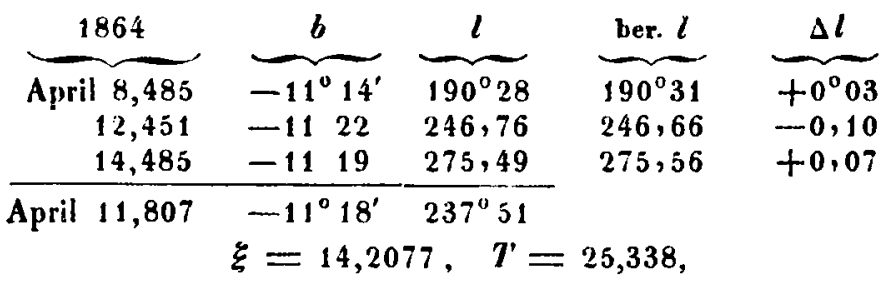

täglich $\Delta \xi=-5^{\prime} 14^{\prime \prime}$; stündlich Oststurm: 6 Meilen.

Durch die verschiedene Bewegung der beiden Flecke Je 44 und 45 wird der gegenseitige Abstand vergrössert. Aus den Beobachtungen folgt der Abstand:

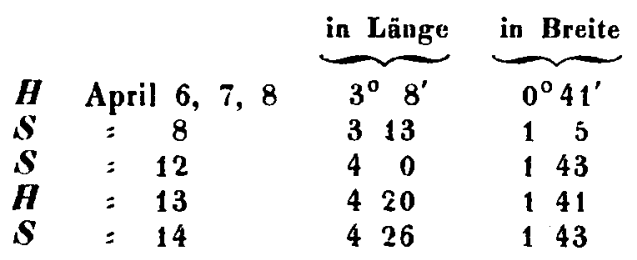

Die Fackeln, welche die beiden Flecke trennten, erlangten also zwischen den Flecken eine grössere Ausdehnung. Ein noch mehr auffallendes Beispiel für die Verbreiterung der Fackeln giebt́ der später zu erwähnende grosse Fleck No 86. Derselbe wurle bei Einschiebung eines Fackelarmes in awei grosse behofte Stücke getrennt, welche sich dann mit zunehmender Breite des Fackelarmes sehr betrichtlich von einander entlernten.
Mo 58 in der Breite $=-5^{\circ} 13^{\prime}$.

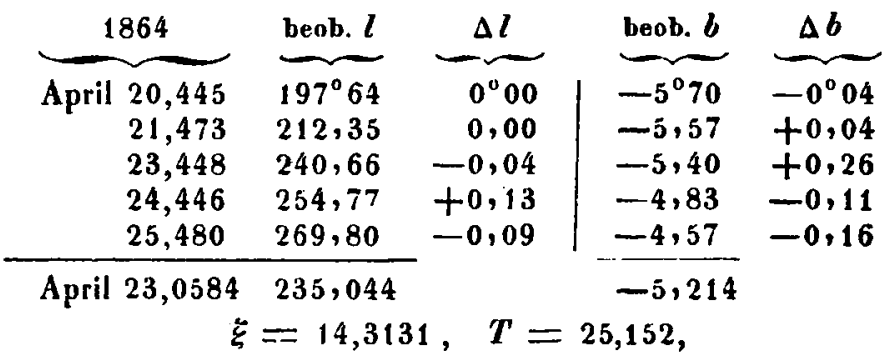

täglich in Länge $=+1^{\prime} 5^{\prime \prime}$; stündlich West : 1 Meile, täglich in Breite dem Aequator zu $=0^{\circ} 12^{\prime}$; stündl. 14 Meil.

To 60 nabe dem Aequator.

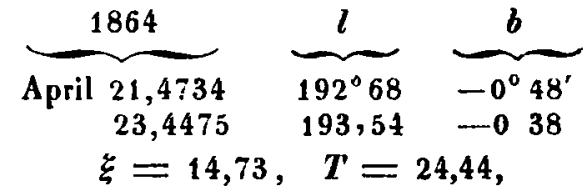

täglich in Länge $=+0^{\circ} 26^{\prime}$; stündlich West : 30 Meilen.

Er näherte sich dem Aequator noch mehr. - Es ist oben bemerkt, dass die Oerter ditses wegen seiner Kleinheit für die Beobachtung sehr günstigen Flecks als besonders genau angesehen werden dürfen, mithin das aus den beiden einander naben Orten hergeleitete Resultat für hinreichend zuverlässig erachtet werden kann.

Auclam, 1864 Nor. 17.

\title{
Beobachtungen und Elemente des Cometen I. 1864.
}

Von Herrn Tebbutt, Director der Sternwarte zu Windsor in New-South-Wales.

I now send you further oliservations and also ny deternination of the orbit of the comet, which forned the subject of my letter to you of the 19. August. The observations, extending from the 14. to the 21. August and from the 4 . to the 17. September were male under the disadvantage of strong moonlight, and on the 15 . September a strong westwind blew into the shutters, which circumstance interfered much with the accuracy of the work on that evening. The observations are corrected for refraction where necessary. The calculation of the orhit has been peculiarly tedious and difficult owing to the observations in the first approximation being near the perihelion, and the comet's radius vector in two of the observations for the second approximation being nearly perpendicular to the visual ray from the earth to the comet. I think, however, the subjoined elements will be found to be a pretty close approximation of the true orbit; the observations for Aug. 14 and 25 and Sept. 4, taking into account the corrections for aberration and parallax, are represented as follows :

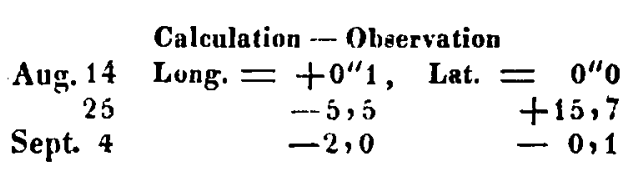

So far as I have been able to learn, the comet was first seen in these colonies by Mr. E. Quaife of Windsor about $6^{\mathrm{h}} 30^{\text {in }}$ on the 10. August, but 1 have no doubt, it was long previously discovered in the observatories of Europe. It is remarkahle for its proximity to the earth during the first week of August, the sinall inclination of its orbit, and its near approach to the earth's orbit at the descending node.

\section{Elements.}

$T^{\prime}=1864$ August 15,59985 Greenwich M. T.

$$
\begin{aligned}
& \pi=304^{\circ} 10^{\prime} 14^{\prime \prime} 9 \\
& \Omega=95832,3\} \\
& i=152 \quad 3,7 \\
& \log q=9,9587215 \text {. } \\
& \text { M. Equin. 1864,0 } \\
& \text { Motion : Retrograde. }
\end{aligned}
$$




\begin{tabular}{|c|c|c|c|c|}
\hline \multirow[b]{2}{*}{1864} & \multirow[b]{2}{*}{ Windsor $\mathbf{M}$. T } & \multicolumn{3}{|c|}{ Comet - Star } \\
\hline & & $\mathbf{A R}$ & N.P & $.1)$ \\
\hline gg. 18 & $8^{h} 40^{m} 42^{s}$ & $-12^{m} 30^{s} 59$ & $+11^{\circ}$ & $28^{\prime \prime} 5$ \\
\hline 19 & $\begin{array}{lll}6 & 39 & 41\end{array}$ & $+020,29$ & & 10,9 \\
\hline 21 & $\begin{array}{llll}6 & 36 & 37\end{array}$ & $-134,88$ & - & 56,8 \\
\hline$=$ & $\begin{array}{lll}8 & 32 & 47\end{array}$ & $-123,36$ & +0 & 1,3 \\
\hline 25 & 63346 & $-212,89$ & -17 & 35,4 \\
\hline$=$ & $\begin{array}{lll}8 & 19 & 58\end{array}$ & $-26,92$ & -16 & 37,2 \\
\hline 26 & 81413 & $-051,79$ & $-\mathbf{j}$ & $2, j$ \\
\hline 27 & $\begin{array}{lll}8 & 12 & 10\end{array}$ & $+016,38$ & +7 & 10,7 \\
\hline 30 & 8 & $+231,71$ & +12 & 36,7 \\
\hline Sept. 2 & $\begin{array}{lll}9 & 15 & 49\end{array}$ & $-25 \quad 7,21$ & +2 & 36,6 \\
\hline 3 & $\begin{array}{lll}8 & 15 & 2\end{array}$ & $-2435,98$ & +8 & 23,8 \\
\hline 4 & $\begin{array}{lll}8 & 16 & 22\end{array}$ & $-24 \quad 5,45$ & +13 & 42,2 \\
\hline 6 & 754 & $-117,15$ & -15 & 16,1 \\
\hline 11 & 7206 & $+032,62$ & +2 & 53,7 \\
\hline 12 & $\begin{array}{lll}7 & 29 & 36\end{array}$ & $+051,31$ & & $\cdots$ \\
\hline 13 & $\begin{array}{lll}7 & 25 & 36\end{array}$ & +1 & +9 & 0,6 \\
\hline 15 & $\begin{array}{lll}7 & 19 & 14\end{array}$ & $+143,06$ & +15 & 0,7 \\
\hline 16 & $\begin{array}{lll}7 & 26 & 6\end{array}$ & $+2451,17$ & +11 & 50,5 \\
\hline 17 & 73220 & $+25 \quad 7,60$ & +14 & 34,6 \\
\hline
\end{tabular}

Mean places of the stars of comparison for 1864.0 .

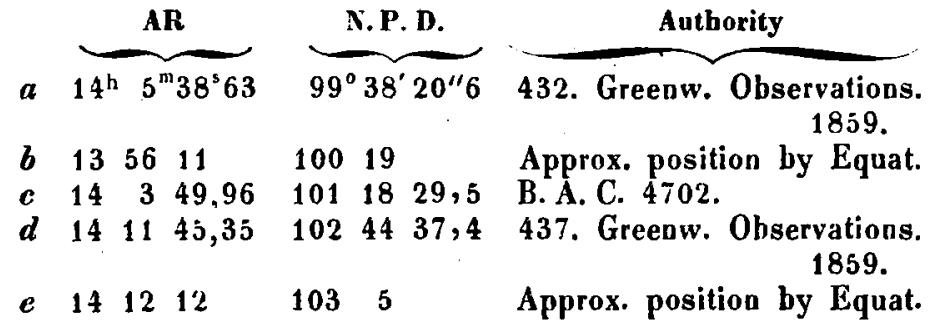

Windsor, New-South-Wales, 1864 Septenber 20.

\begin{tabular}{|c|c|c|c|c|}
\hline \multirow[b]{2}{*}{ Comp. } & \multicolumn{3}{|c|}{ Comet's } & \multirow[b]{2}{*}{ Star } \\
\hline & A pp. & AR & App. N.P.D. & \\
\hline 3 & $13^{h} 53^{r}$ & $10^{\mathrm{s}} 46$ & $99^{\circ} 49^{\prime} 57^{\prime \prime} 4$ & $\boldsymbol{u}$ \\
\hline 6 & $\ldots \ldots$ & $\ldots \ldots$ & $\ldots \ldots \ldots \ldots$ & 6 \\
\hline 4 & 142 & 17,48 & 1011641,5 & $\boldsymbol{c}$ \\
\hline 4 & 142 & 29,00 & 1011839,6 & $c$ \\
\hline 3 & 149 & 34,88 & $10227 \quad 10,5$ & 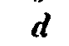 \\
\hline 5 & 149 & 40,85 & $10228 \quad 8,7$ & $d$ \\
\hline 4 & 1410 & 55,97 & 1023943,3 & $d$ \\
\hline 4 & $14 \quad 12$ & 4,12 & 1025156,5 & $d$ \\
\hline 3 & $\ldots \ldots$ & $\ldots \ldots$ & $\ldots \ldots \ldots$ & $e$ \\
\hline 1 & $14 \quad 16$ & 47,28 & 1033732,2 & $f$ \\
\hline 2 & 1417 & 18,50 & $10343 \quad 19,4$ & $f$ \\
\hline 2 & 1417 & 49,02 & 1034837,7 & $f$ \\
\hline 3 & $\ldots \ldots$ & $\cdots$ & $\ldots \ldots \ldots \ldots$ & $g$ \\
\hline 4 & $\ldots \ldots$ & $\cdots \cdots$ & $\ldots \ldots \ldots \ldots$ & I \\
\hline 3 & $\ldots \ldots$ & $\cdots \ldots$ & $\ldots \ldots \ldots$ & $g$ \\
\hline 2 & $\ldots$ & $\ldots \ldots$ & $\ldots \ldots \ldots \ldots$ & $g$ \\
\hline 4 & . & 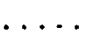 & $\ldots \ldots \ldots \ldots$ & $y$ \\
\hline 1 & 1421 & 58,93 & 1043056,1 & $\pi$ \\
\hline 2 & 1422 & 15,35 & 1043340,1 & $h$ \\
\hline
\end{tabular}

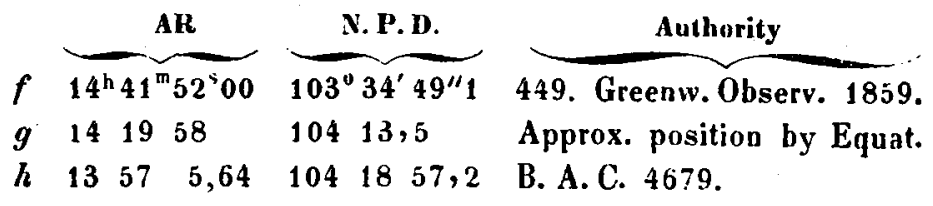

The stars $b, e$ and $g$ are of the 7.magn., $e$ and $g$ are identical with 199 and 347 of Weisse's Catalogue, Hora XIV. Long. of Observatory $=10^{\mathrm{h}} 3^{\mathrm{m}} 20^{\mathrm{s}}$ E. of Greenwich, Lat. = = $=33^{\circ} 36^{\prime} 30^{\prime \prime} \mathrm{S}$.

John Tebbutt junr.

Elemente und Ephemeride der Terpsichore (81). Von Herrn Dr. F. Tietjen.

Da die in den Astr. Nachr. No 1498 gegebenen Elemente des Planeten 81 Terpsichore schon beträcbtlich vom Himmel abweichen, so berechnete ich aus den Berliner Beob. Oct. 6, Oct. 23 und Nov. 13 folgendes neue Elementensystem:

$$
\left.\begin{array}{rl} 
& 1864 \text { Nov. } 13.0 \text { mittl. Berl. Zt. } \\
M & =341^{\circ} 17^{\prime} 46^{\prime \prime} 8 \\
\pi & =481729,6 \\
\Omega & =23145,1 \\
i & =75522,0
\end{array}\right\} \text { mittl. Aeq. 1864,0. }
$$

Die Beobachtung von Nov. 13 ist: $9^{\mathrm{h}} 7^{\mathrm{m}} 14^{\mathrm{s}} \mathrm{m}$. Z. B. Sch. $\alpha=23^{\mathrm{h}} 52^{\mathrm{m}} 47^{\mathrm{s}} 40$, sch. $\delta=+2^{\mathrm{n}} 56^{\prime} 0^{\prime \prime} 3$.

Der Vergleichstern wurde durch Anschluss an mehrere Bessel'sche Sterne hestimmt, und für dessen mittl. Ort 1864 angenommen: $\alpha=23^{\mathrm{b}} 51^{\mathrm{m}} 25^{\mathrm{s}} 94, \delta=+2^{\mathrm{l}} 48^{\prime} 44^{\prime \prime} 4$.

Aus obigen Elementen ergiebt sich nachstehende Ephemeride :

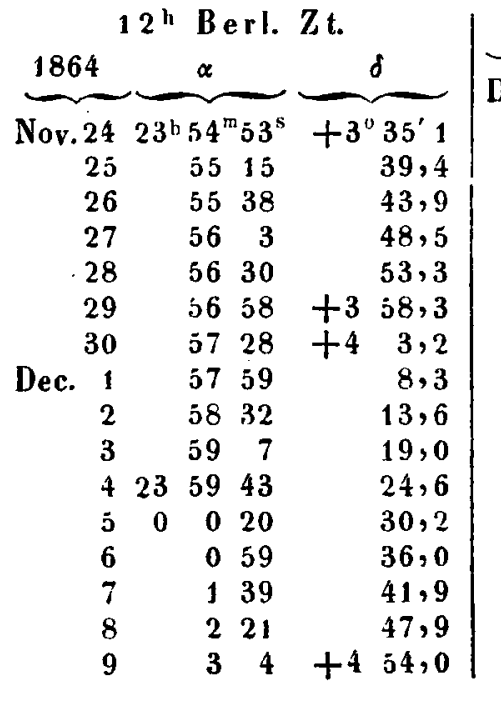

\begin{tabular}{r}
1864 \\
\hline Dec. 10 \\
11 \\
12 \\
13 \\
14 \\
15 \\
16 \\
17 \\
18 \\
19 \\
20 \\
21 \\
22 \\
23 \\
24 \\
25 \\
26
\end{tabular}

Berliu, 1864 Nov. 19.

\begin{tabular}{rrrr}
\multicolumn{1}{c}{$\alpha$} & \multicolumn{1}{c}{$\delta$} \\
$0^{\mathrm{h}} 3^{\mathrm{m}} 48^{\mathrm{s}}$ & $+5^{\mathrm{o}} \boldsymbol{0}^{\prime} 3$ \\
4 & 34 & & 6,7 \\
5 & 21 & & 13,2 \\
6 & 10 & & 19,8 \\
7 & 0 & & 26,5 \\
7 & 51 & & 33,3 \\
8 & 43 & & 40,2 \\
9 & 37 & & 47,2 \\
10 & 32 & +5 & 54,4 \\
11 & 28 & +6 & 1,6 \\
12 & 25 & & 9,0 \\
13 & 24 & & 16,4 \\
14 & 24 & & 24,0 \\
15 & 24 & & 31,6 \\
16 & 26 & & 39,4 \\
17 & 29 & & 47,2 \\
18 & 33 & & 55,1
\end{tabular}

F. Tietjen. 